VIANA MMS; CARLOS LA; SILVA EC; PEREIRA SMF; OLIVEIRA DB; ASSIS MLV. 2015. Composição fitoquímica e potencial antioxidante em hortaliças não convencionais. Horticultura Brasileira 33: 504-509. DOI - http://dx.doi.org/10.1590/S0102-053620150000400016

\title{
Composição fitoquímica e potencial antioxidante de hortaliças não convencionais
}

\author{
Mayara MS Viana ${ }^{1}$; Lanamar A Carlos ${ }^{1}$; Ernani C Silva ${ }^{1}$; Silvia MF Pereira² ${ }^{2}$ Daniela B Oliveira²; Maria \\ LV Assis ${ }^{2}$ \\ ${ }^{1}$ Universidade Federal de São João del Rei, Sete Lagoas-MG, Brasil; mayarasarsur2@yahoo.com.br; lanamar@ufsj.edu.br; clarete@ \\ ufsj.edu.br; ${ }^{2}$ Universidade Estadual do Norte Fluminense, Campos dos Goytacazes-RJ, Brasil; silvia@uenf.br; dbarrosoliveira@uenf.br; \\ lucyassis@gmail.com
}

\begin{abstract}
RESUMO
São denominadas hortaliças não convencionais as espécies vegetais presentes em determinadas localidades ou regiões, mas que aos poucos foram esquecidas e desvalorizadas. Em virtude das mudanças no comportamento alimentar, o consumo destas hortaliças foi reduzido causando perdas econômicas, sociais e culturais. O resgate deste consumo é de grande importância, visando a preservação da biodiversidade brasileira, os benefícios nutricionais, assim como novas fontes de antioxidantes naturais para a indústria alimentícia. Avaliou-se a composição fitoquímica de espécies vegetais denominadas hortaliças não convencionais: beldroega (Portulaca oleracea), bertalha (Basella rubra), caruru (Amaranthus viridis), peixinho (Stachis lanata) e azedinha (Rumex acetosa), bem como o seu potencial alimentar com base no ranquemento do valor dessas características. Com relação aos minerais avaliados, o caruru apresentou os maiores teores de nitrogênio, cálcio, magnésio, enxofre e ferro. O maior conteúdo de compostos fenólicos foi detectado em peixinho, assim como a menor concentração de taninos condensados (0,14 Eag/g de extrato). Quanto aos carotenoides totais, caruru apresentou a maior concentração, mas o conteúdo de $\beta$-caroteno foi maior em peixinho, azedinha tipo I e bertalha. O extrato metanólico de peixinho apresentou forte capacidade de sequestro do radical livre. A maior percentagem de proteína foi detectada na azedinha (tipos I e II). Concluiu-se que os teores de componentes protéicos, minerais e compostos bioativos encontrados nessas hortaliças, caracterizamnas pelo seu elevado potencial nutricional, podendo o seu consumo regular trazer benefícios à saúde humana.
\end{abstract}

Palavras-chave: Portulaca oleracea, Basella rubra, Amaranthus viridis, Stachis lanata, Rumex acetosa, qualidade nutricional.

\begin{abstract}
Phytochemical composition and antioxidant potential of unconventional vegetables

Unconventional vegetable plant species in certain localities or regions, are so called due to have been gradually forgotten and undervaluated. Because of changes in eating behavior, the consumption of these vegetables was reduced causing economic, social and cultural losses. The rescue of this consumption is of great importance to preserve the Brazilian biodiversity, nutritional benefits, as well as new sources of natural antioxidants for the food industry. This study aimed to evaluate the phytochemical composition of plant species called unconventional vegetables: purslane (Portulaca oleracea), bertalha (Basella rubra), pigweed (Amaranthus viridis), peixinho (Stachis lanata) and sorrel (Rumex acetosa), as well as the food potential based on the value of these characteristics. With respect to minerals, pigweed showed the highest levels of nitrogen, calcium, magnesium, sulfur and iron. The highest content of phenolic compounds was detected in peixinho, as well as the lowest concentration of condensed tannins (0,14 Eag/g extract). Regarding total carotenoids, pigweed had the highest concentration, but the content of $\beta$-carotene was higher for peixinho, sorrel type I and bertalha. The methanolic extract of peixinho presented a strong sequestration capacity of free radical. The highest proportion of protein was detected in sorrel (type I and II). The levels of protein, minerals and bioactive compounds found in these unconventional vegetables, characterize these vegetables with high nutritional potential, so that their regular consumption can bring benefits to human health.
\end{abstract}

Keywords: Portulaca oleracea, Basella rubra, Amaranthus viridis, Stachis lanata, Rumex acetosa, nutritional quality.

(Recebido para publicação em 14 de fevereiro de 2014; aceito em 7 de maio de 2015) (Received on February 14, 2014; accepted on May 7, 2015)

\begin{abstract}
$\mathrm{A}$ s hortaliças são plantas alimentícias de grande importância, podendo fornecer desde vitaminas, fibras, carboidratos, minerais até proteínas (MAPA, 2010). Entretanto, o consumo de hortaliças frescas vem diminuindo diante dos hábitos alimentares atuais, resultado da globalização e do consumo exagerado de alimentos industrializados, conver-
\end{abstract}

gindo para uma dieta rica em gorduras e açúcares e reduzida em carboidratos complexos e fibras (Dias et al., 2005). Essas mudanças estão causando perdas nas características alimentares regionais.

As hortaliças não convencionais já foram bastante apreciadas, mas especialmente pela migração da população rural para as cidades grandes e pelo consumo de alimentos de rápido preparo, foram esquecidas e desvalorizadas (Kinupp \& Barros, 2007). Essas hortaliças são popularmente chamadas de "mato" ou "daninhas", mas, podem ser uma alternativa alimentar de qualidade.

Algumas espécies como azedinha (Rumex acetosa), beldroega (Portulaca 
oleracea), bertalha (Basella rubra), caruru (Amaranthus viridis) e peixinho (Stachis lanata) são exemplos destas hortaliças (MAPA, 2010).

Em função da rusticidade, as hortaliças não convencionais são plantas que não necessitam de grandes quantidades de insumos, requerendo menor manejo cultural. Portanto, podem ser utilizadas na agricultura familiar como fonte de renda e de diversificação agrícola, principalmente para populações de baixa renda (Rocha et al., 2008).

Além das propriedades nutricionais e funcionais, as hortaliças não convencionais possuem características sensoriais que facilitam o seu consumo. Como exemplo, as folhas de azedinha podem ser consumidas cruas em saladas ou cozidas sob forma de purê, ou ainda como erva aromática em sopas e temperos (Cunha et al., 2003). As folhas tenras do caruru podem ser utilizadas em saladas e em concentrados proteicos, assim como o espinafre (Wheeker et al., 2006).

Um ponto relevante, quando se trata de hortaliças não convencionais, está relacionado aos teores de minerais os quais são, geralmente, significativamente maiores que em plantas cultivadas (Booth et al., 1992; Guerrero et al., 1998). Estas hortaliças podem apresentar também compostos com função antioxidante (Schmeda-Hirschmann et al., 2005). Os alimentos com função antioxidante possuem substâncias capazes de inibir a oxidação, reduzindo a concentração de radicais livres no organismo. Entre os antioxidantes não enzimáticos estão as vitamina C (ácido ascórbico), vitamina $\mathrm{E}$ (tocoferol), os carotenoides e os flavonóides. Os flavonóides estão incluídos no grupo dos compostos fenólicos (Pieniz et al., 2009).

Estes compostos, além de atuarem na captura de radicais livres, podem estar envolvidos em outros mecanismos fisiológicos que estimulam a atividade das enzimas antioxidantes ou como sinalizadores celulares que ativam e/ou inibem a expressão de algumas enzimas relacionadas com o processo cancerígeno (Shahidi et al., 2007).

Diversos estudos têm demonstrado que o consumo de substâncias antioxidantes na dieta diária pode produzir ação protetora efetiva contra os processos oxidativos que naturalmente ocorrem no organismo (Brenna \& Pagliarini, 2001). Assim, somado aos efeitos protetores das defesas antioxidantes no organismo, o consumo de compostos com capacidade antioxidante através da dieta é de grande importância. Os vegetais, principais fontes de vitaminas antioxidantes, estão associados com o baixo risco de doenças crônicas não transmissíveis (Berger, 2005). Além destas vitaminas, várias outras substâncias com atividade antioxidante podem ser encontradas nos vegetais comumente consumidos na dieta.

Assim, diante das características de biodiversidade, da importância nutricional e ainda pelo resgate cultural associado à carência de informações, bem como a demanda da população por alimentos saudáveis e da indústria alimentícia por fontes naturais de antioxidantes, este trabalho teve como objetivo avaliar a composição fitoquímica de espécies vegetais denominadas de hortaliças não convencionais bem como seu potencial antioxidante e alimentar.

\section{MATERIAL E MÉTODOS}

Foram avaliadas neste estudo as hortaliças não-convencionais: beldroega (Portulaca oleracea), bertalha (Basella rubra), caruru (Amaranthus viridis) peixinho (Stachis lanata) e azedinha (Rumex acetosa). Foram avaliados os tipos I e II de azedinha (Silva et al., 2013), denominadas regionalmente de EMATER e EPAMIG, respectivamente. As plantas do tipo I possuem porte maior em razão de maior peso, maior comprimento e largura do limbo foliar e possuem coloração verde-clara; a azedinha do tipo II possui porte menor e coloração verde-escura (Silva et al., 2013).

O cultivo das hortaliças foi realizado sem o uso de agrotóxico e os tratos culturais foram realizados de acordo com o desenvolvimento das plantas.

As folhas das espécies selecionadas para este estudo foram colhidas no banco de hortaliças não convencionais da Empresa de Pesquisa Agropecuária de Minas Gerais (EPAMIG) do município de Prudente de Morais-MG (19²8'33'S, 44¹1'53'O, altitude 769 m). A colheita das folhas foi realizada ao acaso, em locais em que o material correspondia às folhas jovens e em estado homogêneo de maturação, totalizando $450 \mathrm{~g}$ de cada espécie. Este material foi transportado sob refrigeração para o laboratório e preparado para as devidas análises subsequentes. Parte do material foi congelado em ultrafreezer a $-80^{\circ} \mathrm{C}$ para as análises de carotenoides totais, $\beta$-caroteno e vitamina $C$. Uma parte do material in natura foi triturada com auxílio de um triturador/homogeneizador tipo turrax para execução das análises de $\mathrm{pH}$, ácidez total titulável, umidade, e sólidos solúveis. Finalmente, outra parte das amostras das hortaliças coletadas, foi seca em estufa de circulação forçada de ar, por 48 horas a $65^{\circ} \mathrm{C}$ para análise de compostos fenólicos totais, taninos condensados, composição centesimal e preparação de extratos metanólicos utilizados na análise de atividade antioxidante. Para análise da composição mineral (macro e micronutrientes) foram utilizadas as amostras secas em estufa de circulação forçada de ar à temperatura de $45^{\circ} \mathrm{C}$ por 72 horas.

Foram avaliadas as características físico-químicas: a) teor de sólidos solúveis, determinado com o auxílio de refratômetro digital modelo R2mini, e os resultados expressos em ${ }^{\circ}$ Brix; b) conteúdo de umidade obtido pela diferença entre as massas inicial e final da amostra seca em estufa de esterilização e secagem a $105^{\circ} \mathrm{C}$ por um período de 48 horas (AOAC, 1992); c) pH determinado por potenciometria e d) acidez titulável determinada por titulometria com solução padrão de $\mathrm{NaOH} 0,01 \mathrm{~N}$ utilizando fenolftaleína como indicador (AOAC, 1992) e expressa em gramas de ácido cítrico por grama matéria seca.

Além disso, foram determinados os teores de proteínas, lipídios e cinzas seguindo os protocolos da AOAC (1992). A determinação de fibra detergente neutro (FDN) foi realizada de acordo com Silva \& Queiroz (2002).

Também foram avaliadas a composição mineral dessas hortaliças como nitrogênio $(\mathrm{N})$, determinado pelo método de Nessler (Jackson, 1965); fósforo (P), por colorimetria; potássio $(\mathrm{K})$, por fotometria de chama; cálcio $(\mathrm{Ca})$, magnésio $(\mathrm{Mg})$, ferro $(\mathrm{Fe})$, zinco $(\mathrm{Zn})$, cobre $(\mathrm{Cu})$ 
e manganês (Mn) por espectrofotometria de absorção atômica; e enxofre (S) por turbidimetria com cloreto de bário (Jones Junior et al., 1991; Malavolta et al., 1997).

O teor de ácido ascórbico foi avaliado por cromatografia líquida de alta eficiência (HPLC) da marca Shimadzu, equipado com duas bombas modelo LC-10AD e detector SPD-M10A Diode Array, utilizando-se a técnica descrita por Benlloch et al. (1993) e adaptada por Oliveira Junior et al. (2005).

Os carotenoides totais foram quantificados por espectrofotometria a 450 $\mathrm{nm}$, segundo normas do Instituto Adolfo Lutz (1985) e os resultados expressos em $\mu \mathrm{g}$ de carotenoides por grama de matéria fresca. A concentração de carotenoides totais foi calculada utilizando a seguinte fórmula:

CT $(\mu \mathrm{g}$ carotenoides/g amostra $)=$

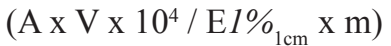

Onde $A=$ absorbância a $450 \mathrm{~nm} ; \mathrm{V}=$ volume final da amostra $(\mathrm{mL}) ; \mathrm{m}=$ massa da $\operatorname{amostra}(\mathrm{g}) ; \mathrm{E} 1 \% 1 \mathrm{~cm}=$ coeficiente de extinção do $\beta$-caroteno em éter de petróleo $=2592$.

$\beta$-caroteno foi quantificado por cromatografia líquida de alta eficiência (HPLC), segundo a metodologia utilizada por Batista et al. (2006) e Rodrigues-
-Amaya et al. (1996). As amostras foram injetadas em HPLC Shimadzu modelo LC 10 AD, provido de "loop" de 20 mL; coluna YMC C30 - Carotenoids (250 $\mathrm{mm}$ de comprimento, 4,6 $\mathrm{mm}$ de diâmetro, $3 \mu \mathrm{m})$, detector UV visível (arranjos de diodos), modelo SPD 10 $\mathrm{AV}$, fase móvel metanol:metil-tert-butil-éter, (65:35); com vazão de $0,8 \mathrm{~mL} /$ minuto e detecção a $450 \mathrm{~nm}$.

Os conteúdos de compostos fenólicos totais e taninos condensados foram quantificados respectivamente pelos métodos espectrofotométricos de Folin-Ciocalteau e do butanol-HCl (Neves et al., 2009). Os resultados obtidos foram expressos em miligramas de equivalente de ácido gálico por (Eag/g) e em mg de catequina (CAE) por grama de extrato.

A atividade antioxidante foi avaliada pelo método espectrofotométrico do radical livre estável DPPH (1,1-difenil-2-picrilidrazila). Este método baseia-se no seqüestro do radical 1,1-difenil-2-picrilhidrazila (DPPH) pelos antioxidantes, que produzem redução de absorção em $515 \mathrm{~nm}$. Quando uma solução de DPPH é misturada com uma substância que pode doar um átomo de hidrogênio, a forma reduzida do radical gerado é acompanhada de perda de cor (Ali et al., 2009). A atividade sequestradora de radicais livres foi calculada pela fórmula
\% Inibição $\left.=\left[\mathrm{A}_{\mathrm{B}}-\mathrm{A}_{\mathrm{A}}\right) / \mathrm{A}_{\mathrm{B}}\right] \times 100$

onde $A_{B}=$ absorção da amostra controle; $A_{A}=$ absorção do extrato testado.

\section{RESULTADOS E DISCUSSÃO}

O caruru apresentou a maior média de sólidos solúveis $\left(17,49^{\circ}\right.$ Brix $)$ e a bertalha a menor $\left(3,14^{\circ}\right.$ Brix) (Tabela 1$)$. Este teor é próximo ao encontrado para rúcula $\left(3,3^{\circ} \mathrm{Brix}\right)$, conforme descrito por Sigrist (2002).

Os valores de umidade variaram de $83,19 \%$ para bertalha a $61,67 \%$ para caruru (Tabela 1). Estes resultados corroboram que a água é o maior componente das hortaliças, variando de 80 a 95\% de sua composição (Chitarra \& Chitarra, 2005).

Todas as hortaliças apresentaram valores de $\mathrm{pH}$ inferiores a 7 (Tabela 1). Silva et al. (2013) encontraram para as duas azedinhas tipo I e II valores de 3,22 e 2,05 , respectivamente.

Os valores de acidez titulável variaram de $0,11 \mathrm{~g}$ ácido cítrico/100 g de massa fresca para bertalha a 0,27 $\mathrm{g}$ ácido cítrico/100 g de massa fresca para peixinho (Tabela 1). Os valores de acidez titulável observados por Silva et al. (2013) para os dois tipos de azedinha foram: tipo I, 1,26 g ácido cítrico/100 g

Tabela 1. Características físico-químicas: teor de sólidos solúveis (SS), umidade (UD), potencial hidrogeniônico (pH) e acidez titulável (AT) avaliados nas hortaliças não convencionais com seus respectivos desvios-padrão (S) \{physico-chemical characteristics: soluble solids content (SS), moisture (UD), hydrogen potential ( $\mathrm{pH}$ ) and titratable acidity (AT) evaluated in unconventional vegetables with their respective standard deviations $\}$. Sete Lagoas, UFSJ, 2013.

\begin{tabular}{lcccccccc}
\hline Hortaliça (folha) & SS $\left({ }^{\mathbf{0}}\right.$ Brix) & $\mathbf{S}$ & UD (\%) & $\mathbf{S}$ & $\mathbf{p H}$ & $\mathbf{S}$ & $\begin{array}{c}\text { AT (g ác. cítrico/100 } \\
\text { g de massa fresca) }\end{array}$ & $\mathbf{S}$ \\
\hline Beldroega & 4,47 & 0,31 & 77,92 & 1,12 & 5,02 & 0,78 & 0,14 & 0,02 \\
Bertalha & 3,14 & 0,41 & 83,19 & 1,16 & 5,85 & 0,89 & 0,11 & 0,05 \\
Caruru & 17,49 & 1,14 & 61,67 & 0,89 & 6,72 & 0,93 & 0,22 & 0,01 \\
Peixinho & 8,20 & 0,83 & 70,72 & 2,01 & 6,11 & 0,90 & 0,27 & 0,03 \\
\hline
\end{tabular}

Tabela 2. Teores de macro e micronutrientes detectados nas hortaliças não convencionais: nitrogênio $(\mathrm{N})$, fósforo $(\mathrm{P})$, potássio $(\mathrm{K})$, cálcio $(\mathrm{Ca})$, magnésio $(\mathrm{Mg})$, enxofre $(\mathrm{S})$, ferro $(\mathrm{Fe})$, manganês $(\mathrm{Mn})$, cobre $(\mathrm{Cu})$, zinco $(\mathrm{Zn})$ e boro $(\mathrm{B})$ \{levels of macro and micronutrients detected in unconventional vegetables: nitrogen $(\mathrm{N})$, phosphorus $(\mathrm{P})$, potassium $(\mathrm{K})$, calcium $(\mathrm{Ca}), \operatorname{magnesium}(\mathrm{Mg})$, sulfur $(\mathrm{S})$, iron $(\mathrm{Fe})$, manganese $(\mathrm{Mn})$, copper $(\mathrm{Cu})$, zinc $(\mathrm{Zn})$ and boron $(\mathrm{B})\}$. Sete Lagoas, UFSJ, 2013.

\begin{tabular}{|c|c|c|c|c|c|c|c|c|c|c|c|}
\hline \multirow{2}{*}{ Hortaliça } & \multicolumn{6}{|c|}{ Macronutrientes (mg/g) } & \multicolumn{5}{|c|}{ Micronutrientes (mg/g) } \\
\hline & $\mathbf{N}$ & $\mathbf{P}$ & $\mathbf{K}$ & $\mathbf{C a}$ & Mg & $\mathbf{S}$ & $\mathrm{Fe}$ & Mn & $\mathrm{Cu}$ & $\mathbf{Z n}$ & B \\
\hline Beldroega & 28,6 & 5,3 & 53,7 & 14,0 & 8,4 & 2,8 & 188,6 & 22,0 & 14,0 & 126,3 & 41,0 \\
\hline Bertalha & 8,3 & 3,6 & 19,5 & 10,5 & 4,8 & 1,2 & 112,5 & 16,5 & 8,2 & 10,4 & 33,1 \\
\hline Caruru & 34,9 & 4,4 & 29,9 & 32,0 & 8,6 & 3,0 & 790,0 & 17,3 & 5,9 & 27,5 & 21,1 \\
\hline Peixinho & 22,8 & 7,1 & 22,7 & 10,7 & 2,5 & 1,4 & 135,0 & 46,8 & 6,9 & 22,4 & 39,6 \\
\hline
\end{tabular}


Tabela 3. Teores de fenóis totais (FT, mg de Eag/g de extrato); taninos condensados (TC, mg de CAT/g de extrato); vitamina C (Vit C, $\mathrm{mg} / \mathrm{g}$ de massa seca); carotenóides totais (CT, $\mu \mathrm{g} / \mathrm{g}$ de massa fresca) e $\beta$-caroteno ( $\beta$-C), $\mu \mathrm{g} / \mathrm{g}$ de massa fresca) detectados nas hortaliças não convencionais com seus respectivos desvios-padrão (S) \{levels of total phenols (FT, mg of Eag/g of extract), condensed tannins (TC, mg of $\mathrm{Eag} / \mathrm{g}$ of extract), vitamin C (Vit C, $\mathrm{mg} / \mathrm{g}$ of dry mass) and total carotenoids (CT, $\mu \mathrm{g} / \mathrm{g}$ of fresh mass) detected in unconventional vegetables and $\beta$-caroten $(\beta-\mathrm{C}), \mu \mathrm{g} / \mathrm{g}$ of fresh mass) with their respective standard deviations (S) $\}$. Sete Lagoas, UFSJ, 2013.

\begin{tabular}{lcccccccccc}
\hline Hortaliça & FT & S & TC & S & Vit.C & S & CT & S & $\boldsymbol{\beta}-\mathbf{C}$ & S \\
\hline Azedinha (tipo I) & 0,23 & 0,02 & 5,56 & 0,85 & *nd & & 95,64 & 1,12 & 7,81 & 0,88 \\
Azedinha (tipo II) & 0,35 & 0,08 & 2,62 & 0,08 & 72,45 & 2,08 & 83,85 & 1,34 & *nd & \\
Bertalha & 0,25 & 0,05 & 0,33 & 0,07 & n.d & & 83,73 & 1,67 & 7,36 & 1,01 \\
Beldroega & 0,40 & 0,10 & n.d & & n.d & & 70,49 & 1,98 & 1,05 & 0,08 \\
Caruru & 0,56 & 0,11 & 1,64 & 0,06 & n.d & & 192,77 & 2,12 & 1,71 & 0,12 \\
Peixinho & 0,77 & 0,12 & 0,14 & 0,01 & n.d & & 103,24 & 1,98 & 7,84 & 0,13 \\
\hline
\end{tabular}

*nd= não determinado.

Tabela 4. Capacidade antioxidante (\%) dos extratos metanólicos das folhas de hortaliças não convencionais e dos padrões testados: quercetina (QT), rutina (RT), ácido ascórbico (ACA) e butil-hidroxi-tolueno (BHT) com seus respectivos desvios-padrão (S) \{antioxidant capacity (\%) of methanol extracts of the leaves of unconventional vegetables and patterns tested: quercetin (QT), rutin (RT), ascorbic acid (ACA) and butyl hydroxy toluene (BHT) with their respective standard deviations (S)\}. Sete Lagoas, UFSJ, 2013.

\begin{tabular}{|c|c|c|c|c|c|c|}
\hline \multirow{2}{*}{ Hortaliça } & \multicolumn{6}{|c|}{ Atividade antioxidante do extrato metanólico (\%) } \\
\hline & $1,00(\mathrm{mg} / \mathrm{mL})$ & $\mathbf{S}$ & $0,10(\mathrm{mg} / \mathrm{mL})$ & $\mathbf{S}$ & $0,01(\mathrm{mg} / \mathrm{mL})$ & $\mathbf{S}$ \\
\hline Azedinha tipo I & 69,23 & 1,15 & 62,53 & 1,55 & 12,43 & 0,12 \\
\hline Azedinha tipo II & 61,00 & 2,01 & 59,86 & 1,19 & 9,31 & 0,09 \\
\hline Bertalha & 60,00 & 1,98 & 40,86 & 1,01 & 13,49 & 0,12 \\
\hline Beldroega & 63,25 & 1,01 & 45,36 & 1,02 & 12,62 & 0,15 \\
\hline Caruru & 47,37 & 0,88 & 26,07 & 0,68 & 11,76 & 0,91 \\
\hline \multirow[t]{2}{*}{ Peixinho } & 99,09 & 1,76 & 89,90 & 2,10 & 26,50 & 1,10 \\
\hline & \multicolumn{6}{|c|}{ Padrões } \\
\hline QT & 100,00 & & 95,27 & & 91,27 & \\
\hline RT & 88,00 & & 86,42 & & 0,85 & \\
\hline ACA & 95,63 & & 93,33 & & 92,73 & \\
\hline BHT & 100,00 & & 52,10 & & 43,60 & \\
\hline
\end{tabular}

Tabela 5. Teores de lipídios, cinzas, proteína e fibras em hortaliças não convencionais com seus respectivos desvios-padrão (S) \{relative values of lipids, ash, protein and fibers in unconventional vegetables with their respective standard deviations (S)\}. Sete Lagoas, UFSJ, 2013.

\begin{tabular}{lcccccccc}
\hline Hortaliça & Lipídeos (\%) & S & Cinzas (\%) & S & Proteína (\%) & S & Fibras (FDN, \%) & S \\
\hline Azedinha tipo I & 2,16 & 0,08 & 16,67 & 1,01 & 29,34 & 0,02 & 30,34 & 0,55 \\
Azedinha tipo II & 2,03 & 0,07 & 15,92 & 1,02 & 27,49 & 1,14 & 33,69 & 0,54 \\
Bertalha & 1,37 & 0,10 & 4,33 & 1,08 & 17,44 & 1,56 & 31,75 & 0,56 \\
Beldroega & 3,75 & 0,08 & 20,56 & 2,10 & 12,82 & 1,00 & 36,27 & 1,12 \\
Caruru & 1,92 & 0,07 & 20,11 & 2,15 & 25,61 & 1,13 & 40,04 & 0,78 \\
Peixinho & 2,62 & 0,05 & 9,95 & 0,81 & 24,01 & 1,16 & 45,85 & 0,78 \\
\hline
\end{tabular}

de massa fresca e tipo II, 2,23 g ácido cítrico/100 g de massa fresca correlacionando assim aos seus baixos valores de $\mathrm{pH}$. A acidez indica a presença de ácidos orgânicos nos vegetais. Com algumas exceções, as hortaliças apresentam baixa acidez, sendo portanto suscetíveis à deterioração por bactérias
(Paschoalino, 1997).

Em termos de teores de macro e micronutrientes, encontrou-se para o caruru as maiores médias de $\mathrm{Ca}, \mathrm{Mg}, \mathrm{N}$ e S e teor bastante elevado de $\mathrm{Fe}(790 \mathrm{~g} /$ kg) (Tabela 2). Segundo Franco (2004), a recomendação diária de ferro é de 10 mg para homens e $15 \mathrm{mg}$ para mulheres.
Comparando-se as quantidades de minerais encontrados em todas as hortaliças não convencionais estudadas com os valores estabelecidos para as necessidades diárias de ferro verificou-se que essas hortaliças podem contribuir significativamente com a nutrição humana, se consumidas no cotidiano. 
Os maiores teores de fósforo foram encontrados em peixinho $(7,1 \mathrm{mg} / \mathrm{g}) \mathrm{e}$ beldroega $(5,3 \mathrm{mg} / \mathrm{g})$. Dentre as hortaliças presentes na tabela brasileira de composição de alimentos (NEPA/ UNICAMP, 2013), o agrião apresentou $8,5 \mathrm{mg} / \mathrm{g}$, a couve-flor $7,2 \mathrm{mg} / \mathrm{g}$ e o inhame $2,4 \mathrm{mg} / \mathrm{g}$ de fósforo, resultados próximos aos encontrados neste estudo. Os valores de enxofre encontrados em caruru $(3,0 \mathrm{mg} / \mathrm{g})$ e beldroega $(2,8$ $\mathrm{mg} / \mathrm{g}$ ) são próximos aos encontrados em couve $(3,06 \mathrm{mg} / \mathrm{g})$ e repolho $(3,24 \mathrm{mg} / \mathrm{g})$ (Franco, 2004).

Os compostos fenólicos compõem uma das maiores classes de metabólitos em vegetais, ocorrendo em diferentes tecidos e alimentos. Fazem parte desse grupo os ácidos fenólicos, taninos e flavonóides. Relatos sobre as ocorrências de compostos fenólicos refletem a atividade antioxidante observada para as fontes vegetais (Hassimoto et al., 2005), capaz de prevenir inúmeras doenças crônicas não transmissíveis em seres humanos.

Não foi detectada a presença de taninos condensados na beldroega; as demais hortaliças apresentaram quantidades apreciáveis (Tabela 3) desse composto fenólico. De acordo com Degáspari et al. (2005), a presença de pequenas quantidades de taninos confere características sensoriais desejáveis ao alimento, no entanto, quantidades apreciáveis (Tabela 3) conferem características adstringentes. A sensação de adstringência é gerada devido à propriedade que os taninos apresentam de precipitar proteínas.

Com relação ao conteúdo de vitamina $C$, a azedinha tipo II apresentou 72,45 $\mathrm{mg}$ de vitamina C/grama de massa seca. Nas demais amostras não foi detectada a presença de vitamina C (Tabela 3). De acordo com Pilon (2003), a vitamina C é a mais instável das vitaminas por ser sensível a agentes como luz, oxigênio e calor.

Detectou-se para o caruru o maior teor de carotenoides em relação às demais hortaliças (Tabela 3). Este pigmento é sensível à luz, à temperatura e à acidez (Ramos et al., 2011). Por isso as hortaliças devem ser ingeridas de preferência, logo após a colheita, devendo mantê-las armazenadas por um menor período possível.

As hortaliças azedinha (tipo I), bertalha e peixinho apresentaram os maiores valores de $\beta$-caroteno (Tabela 3). O $\beta$-caroteno, também conhecido como pró-vitamina $\mathrm{A}$, é um pigmento que possui coloração laranja, também sensível à luz e ao oxigênio. Este pigmento está associado à proteção contra doenças cardiovasculares e câncer (Carvalho et al., 2006).

Em valores absolutos, o extrato metanólico de peixinho exibiu forte capacidade de sequestro do radical DPPH nas concentrações de $1 \mathrm{mg} / \mathrm{mL}$ e 0,1 $\mathrm{mg} / \mathrm{mL}$, sendo semelhante à capacidade antioxidante do BHT (hidroxitolueno de butilato) e da quercetina (Tabela 4). $\mathrm{Na}$ maior concentração ( $1 \mathrm{mg} / \mathrm{mL})$, exibiu atividade superior ao BHT e semelhante ao da quercetina evidenciando o potencial antioxidante deste extrato.

Os extratos das azedinhas (tipo I e II) apresentaram capacidade de sequestro do DPPH, superior a $60 \%$ na maior concentração, sendo que na concentração de $0,1 \mathrm{mg} / \mathrm{mL}$ os extratos tiveram atividades superiores ao BHT, mas inferiores à quercetina, considerando os valores absolutos. Na menor concentração $(0,01 \%)$, todos os extratos avaliados apresentaram capacidade de sequestro do DPPH superiores ao da rutina e inferiores aos demais padrões avaliados nas mesmas condições.

Os maiores teores de proteínas foram $29,34 \%$ na azedinha tipo I, $27,49 \%$ na azedinha tipo II e 25,61\% no caruru (Tabela 5). Estes valores são próximos aos encontrados na Tabela Brasileira de Composição de Alimentos (TACO) para espécies convencionais como a mostarda $(28,57 \%)$ e espinafre $(33,33 \%)$.

Os valores de lipídios encontrados, conforme já esperado, foram baixos (Tabela 5), visto que as hortaliças não são consideradas fonte de óleo (Pinto et al., 2001). Já os teores de cinzas variaram amplamente de 4,33\% para bertalha a $20,56 \%$ para beldroega (Tabela 5). Os teores de fibra detergente neutro (FDN) encontrados variaram de $30,34 \%$ em azedinha tipo I a 45,85\% em peixinho (Tabela 5). Considerando estes valores, as folhas das hortaliças não convencionais apresentaram teores representativos de fibras dietéticas, de acordo com Spiller \& Shipley (2001), citados por Maffia (1991). Os autores apresentaram o conteúdo aproximado de fibra detergente neutro em alguns alimentos, como milho integral (13\%), aveia integral (31\%), farelo de aveia integral (45\%) e farelo de arroz (24\%). Com base nestes dados, as hortaliças não convencionais apresentam quantidade de FDN similar à aveia integral e ao farelo integral.

Concluiu-se que os teores de proteínas, carotenoides, minerais e compostos fenólicos encontrados nas hortaliças não convencionais relacionadas no presente estudo caracterizam esses vegetais como possuidores de elevado potencial nutricional e antioxidante, podendo seu consumo regular estar associado a benefícios à saúde humana.

\section{AGRADECIMENTOS}

Os autores agradecem à Fundação de Amparo à Pesquisa do Estado de Minas Gerais e ao Conselho Nacional de Desenvolvimento Científico e Tecnológico (CNPq 559678/2010-0) e à UFSJ (Universidade Federal de São João del-Rei) pelo apoio financeiro para esta pesquisa e à Empresa de Pesquisa Agropecuária de Minas Gerais (EPAMIG) pelo fornecimento das hortaliças.

\section{REFERÊNCIAS}

ALI SS; KASOJU N; LUTHRA A; SINGH A; SHARANABASAVA H; SAHU A; BORA U. 2009. Indian medicinal herbs as sources of antioxidants. Food Research International 41: 1-15.

AOAC, ASSOCIATION OF OFFICIAL ANALYTICAL CHEMISTS. 1992. Official methods of analysis of the Association of Official Analytical Chemistry. 12. ed. Washington: DC: AOAC, 1140p.

BATISTA MA; PINHEIRO-SANT'ANA HM; PAES JB. 2006. Carotenos e provitamina A em bertalha e ervas aromáticas comercializadas em Viçosa-MG, durante as quatro estações do ano. Acta Sci. Health Sci. 28: 93-100.

BENLLOCH R; FARRÉ R; FRIGOLLA A. 1993. A quantitative estimate of ascorbic and isoascorbic acid by high performance liquid chromatography: application to citric juices. Journal of Liquid Chromatography 16: 3113-3122.

BERGER MM. 2005. Can oxidative damage be treated nutritionally? Clin Nutr. 24: 172-183. 
BOOTH S; BRESSANI R; JOHNS T. 1992. Nutrient content of selected indigenous leafy vegetables consumed by the Kekchi people of Alta Verapaz, Guatemala. Journal of Food Composition and Analysis 5: 25-34.

BRENNA OV; PAGLIARINI E. 2001 Multivariate analyses of antioxidant power and polyphenolic composition in red wines. Journal of Agricultural and Food Chemistry 49: 4841-4844.

CARVALHO PGB; MACHADO CMM; MORETTI CL; FONSECA MEN. 2006. Hortaliças como alimentos funcionais. Horticultura Brasileira 24: 397-404.

CHITARRA MIF; CHITARRA AB. 2005. Póscolheita de frutas e hortaliças: Fisiologia e manuseio. Lavras, ESAL/FAEPE 783p.

CUNHA AP; SILVA AP; ROQUE OR. 2003. Plantas e produtos vegetais em fitoterapia. Lisboa: Fundação Calouste Gulbenkian, p. 152-53.

DEGÁSPARICH; WASZCZYNSKYJN; PRADO MRM. 2005. Atividade antimicrobiana de Schinus terebinthfolius . Ciências Agrotécnica 29: 617-622.

DIAS ACP; PINTO NAVD; YAMADA LTP; MENDES KL; FERNANDES AG. 2005. Avaliação do consumo de hortaliças não convencionais pelos usuários das unidades do Programa Saúde da Família (PSF) de Diamantina-MG. Alim. Nutr. 16: 279-284.

FRANCO G. 2004. Tabela de composição química dos alimentos. São Paulo: Atheneu. 307p.

GUERRERO JLG, MARTÍNEZ JJG., ISASA MGT. 1998. Mineral nutrient composition of edible wild plants. Journal of Food Composition and Analysis 11: 322-328.

HASSIMOTO NMA; GENOVESE MI; LAJOLO FM. 2005. Antioxidant activity of dietary fruits, vegetables, and commercial frozen fruit pulps. Jounal of Agricultural and Food Chemistry 53: 2928-2935.

IAL, INSTITUTO ADOLF LUTZ. 1985. Normas analiticas do Instituto Adolfo Lutz, 3. ed., São Paulo: IAL, 533p.

JACKSON ML. 1965. Soil chemical analysis. New Jersey: Prentice Hall, 498p.
JONES JUNIOR JB; WOLF B; MILLS HA. 1991. Plant analysis handbook: a practical sampling, preparation, analysis, and interpretation guide. Athens: Micro-Macro Publishing. 213p.

KINUPP VF; BARROS IBI. 2007. Riqueza de plantas alimentícias não-convencionais na região metropolitana de Porto Alegre-RS. Revista Brasileira de Biociências 5: 63-65.

MAFFIA UCC. 1991. Avaliação do farelo de arroz em substituição à farinha de trigo na panificação. Viçosa: UFV. 122p. (Dissertação mestrado).

MALAVOLTA E; VITTI GC; OLIVEIRA SA. 1997. Avaliação do estado nutricional das plantas: princípios e aplicações. Piracicaba: POTAFOS, 319p.

MAPA. 2010. Manual de hortaliças nãoconvencionais. Brasília: Mapa/ACS, 92 p.

NEPA/UNICAMP. 2013. Tabela brasileira de composição de alimentos (TACO), versão 2. Disponível em: http://www.unicamp.br/nepa/ taco $>$. Acessado em 25 setembro 2013.

NEVES LC; ALENCAR SM; CARPES ST. 2009. Determinação da atividade antioxidante e do teor de compostos fenólicos e flavonoides totais em amostras de pólen apícola de Apis melifera. J. Food Technol 15: 107-110.

OLIVEIRA JUNIOR LF; CORDEIRO CAM; KONDA L; COELHO EM; CARLOS LA; ARAÚJO TMR. 2005. Vida de prateleira de goiaba minimamente processada submetida a diferentes temperaturas de armazenamento. Revista Brasileira de Armazenamento 30: 157-162.

PASCHOALINO JE. 1997. Hortaliças acidificadas em conserva: riscos e cuidados. Informativo Fruthotec 3: 1-7.

PIENIZ S; COLPO E; OLIVEIRA VR; ESTEFANEL V. 2009. Avaliação in vitro do potencial antioxidante de frutas e hortaliças. Ciência e Agrotecnologia 33: 552-559.

PILON L. 2003. Estabelecimento da vida útil de hortaliças minimamente processadas sob atmosfera modificada e refrigeração. Piracicaba: ESALQ. 111p. (Dissertação mestrado).

PINTO NAVD; FERNANDES SM; THÉ PMP;
CARVALHO VD. 2001. Variabilidade da composição centesimal, vitamina $\mathrm{C}$, ferro e cálcio de partes da folha de taioba. Revista. Brasileira de Agrociência 7: 205-208.

RAMOS DD; VIEIRA MC; FORMAGIO ASN; CARDOSO CAL; RAMOS DD; CARNEVALI TO. 2011. Atividade antioxidante de Hibiscus sabdariffa em função do espaçamento entre plantas. Ciência Rural 41: 1331-1336.

ROCHA DRC; PEREIRA-JÚNIOR GA; PANTOJA L; SANTOS AS; PINTO NAVD. 2008. Macarrão adicionado de ora-pro-nobis (Pereskia aculeata) desidratado. Alimentos e Nutrição 14: 459-465.

RODRIGUEZ DB; RAYMOND LC; LEE T; SIMPSON KL; CHICHESTER CO. 1996. Carotenoid pigment changes in ripening Momordica charantia fruits. Ann. Bot. 40: 615-624.

SCHMEDA-HIRSCHMANN G; FERESIN G; TAPIO A; HILGET A. 2005. Proximate composition and free radical scavenging activity of edible fruits from the Argentina Yungas. Journal of the Science of Food and Agriculture 85: 1357-1364.

SIGRIST JM. 2002. Estudos fisiológicos e tecnológicos de couve-flor e rúcula minimamente processadas. Piracicaba: ESALQ. 112p. (Tese doutorado).

SILVA DJ; QUEIROZ AC. 2002. Análise de alimentos (métodos químicos e biológicos). Viçosa: UFV. 235p.

SILVAEC; CARLOS LA; ARAÚJOAP; FERRAZ LCL; PEDROSA MW; SILVA LS. 2013. Characterization of two types of azedinha in the region of Sete Lagoas, Brazil. Horticultura Brasileira 31: 328-331.

SHAHIDI F; ALASALVAR C; LIYANAPATHIRANA CM 2007. Antioxidant phytochemicals in hazelnut kernel (Corylus avellana) and hazelnut byproducts. Journal of Agricultural and Food Chemistry 55: 1212-1220.

WHEEKER EL; BECKER R; LORENZ K; STAFFORD AE; GROSJEAN OK; BETSCHART AA; SAUNDERS RM. 2006. A composition study of amaranth grain. Journal of Food Science 46: 3630-3634. 